\title{
Management of Thyroid Disease in Pregnancy: Experience from an Antenatal Thyroid Clinic
}

\section{Introduction}

Thyroid disease is common in pregnancy: American data shows that 2-3\% of pregnancies have elevated TSH levels with $0.3-0.5 \%$ diagnosed as hypothyroid and $2-2.5 \%$ affected by subclinical hypothyroidism ${ }^{(1)}$. Management of thyroid disease (with hypothyroidism being most common) in pregnancy is important due to the potential adverse maternal and foetal effects, including neurocognitive disorders, low birth weight and foetal loss ${ }^{(2)}$. Current recommendations by NICE state that clinicians should immediately increase thyroxine dose in hypothyroidism with pregnancy and refer to a specialist, with dose adjustment dependent on initial TSH levels ${ }^{(2)}$. Follow up in both primary and secondary care is vital due to the high probability of thyroid disease in any following pregnancy ${ }^{(3,4)}$.
Micha Thomas ${ }^{1,2}$

Zaki Hassan-Smith ${ }^{1,2}$

Andrew Bates ${ }^{1}$

Asad Rahim ${ }^{1}$

${ }^{1}$ Birmingham Heartlands Hospital

${ }^{2}$ University of Birmingham Medical School

\section{Aim}

This retrospective audit aimed to determine the aetiology of thyroid disease and subsequent management in patients attending an antenatal thyroid clinic ran by one Consultant Endocrinologist at Heartlands Hospital, West Midlands. By reviewing practice, the objectives were to find the frequency of dose changes and the magnitude dose change (\%) for patients attending both live and virtual clinics. Evaluating these changes against current recommendations set by NICE was aimed to review practice and set future recommendations.

\section{Method}

Using electronic records and patient case notes, I searched for patients seen between September 2013 and November 2014 in the antenatal thyroid clinic. 114 women were identified and baseline demographics, biochemical and therapeutic characteristics were collected. Data collection using the pro forma included age, median gestation and dose of treatment at first review, previous disease or co-morbidities, aetiology and any further changes in dose on following reviews until present.

Results found the women had a mean age of 31 years (+/- 5 years). Blood tests were performed at 5-7 week intervals after the initial appointment in the clinic, with a virtual clinic system in place to monitor blood results.

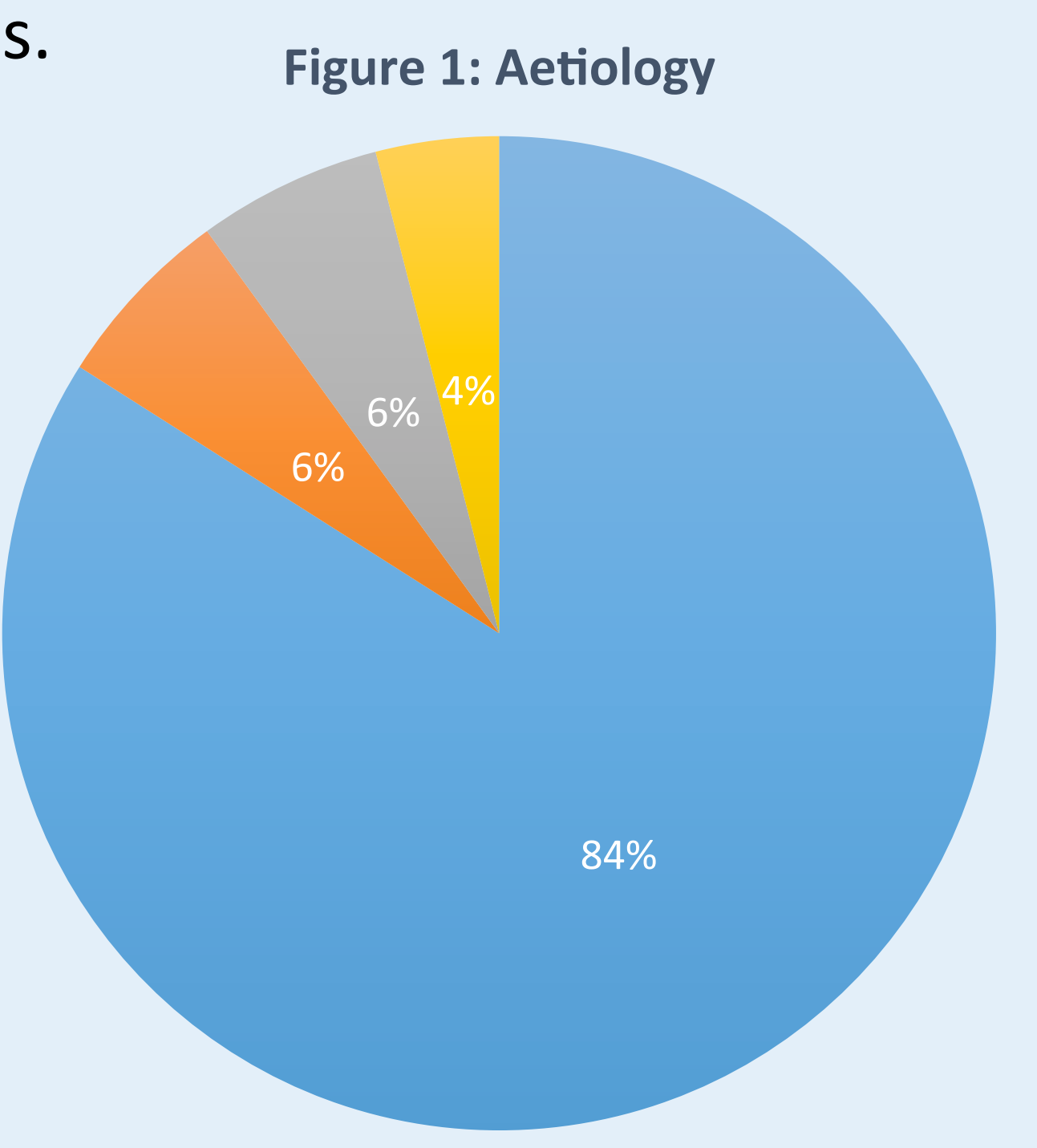

Results

- Median gestation at initial review: 16 weeks (IQR 13-20)

- $89 \%$ patients had previous episodes/ current thyroid disease with mean duration of 5 years (IQR 2.4-8.0)

- $11 \%$ patients had new onset thyroid disease, majority being gestational thyrotoxicosis or positive TPO antibodies but normal function

\section{- Diagnoses:}

\section{a) Hypothyroidism (84\%)}

- Median thyroxine dose at initial consultation $=100 \mathrm{mcg} /$ day $($ IQR 50-144)

\begin{tabular}{|c|c|c|}
\hline $\begin{array}{c}\text { Number dose } \\
\text { adjustments }\end{array}$ & $\begin{array}{c}\text { Number patients } \\
\text { (\% unless stated) }\end{array}$ & TSH (mU/L) \\
\hline 0 & 44 & $1.0+/-1.9$ \\
\hline 1 & 37 & $5.4+/-6.8$ \\
\hline 2 & 13 & $8.2+/ 16.1$ \\
\hline 3 & 5 & $4+/-0.22$ \\
\hline 4 & 1 patient & $<0.01$ \\
\hline
\end{tabular}

b)Gestational thyrotoxicosis (6\%) conservative management

c)Grave's disease (6\%) - no adverse foetal/ maternal outcomes, no new-onset Grave's seen

\section{d)Other (4\%)}

NB: Mean +/- SD for TSH is quoted at time dose adjustment was necessary

- On final follow up, FT4 had normalised in all hypothyroid patients

- Only one patient had a mild elevation of TSH at $7.2 \mathrm{mU} / \mathrm{L}$

- There were no adverse effects of treatment described in the data

\section{References}

1) The American Thyroid Association Taskforce on Thyroid Disease During Pregnancy and Postpartum, Alex Stagnaro-Green, Marcos Abalovich, Erik Alexander, Fereidoun Azizi, Jorge Mestman, Roberto Negro, Angelita Nixon, Elizabeth N. Pearce, Offie P. Soldin, Scott Sullivan, and Wilmar Wiersinga. Thyroid. October 2011, 21(10): 1081-1125. doi:10.1089/thy. 2011.0087 $\mathrm{N}$ I C E C K S. H y p o t h y r o i d i $s$ m. http://cks.nice.org.uk/ hypothyroidism\#!references/-481160 (last accessed 01/10/15)

BTA, ACB and BTF (2006) UK quidelines for the use of thyroid function tests. British Thyroid Association, Association for Clinical Biochemistry, British Thyroid Foundation. www.british-thyroid-association.org (last accessed 01/10/15) The Endocrine societ

1 The Endocrine Society. Management of Thyroid Dysfunction during Guideline. J Clin Endocrinol Metab, 97: 2543-2565. 2012.
Figure 2: Dose Adjustments required in the Management of Hypothyroidism

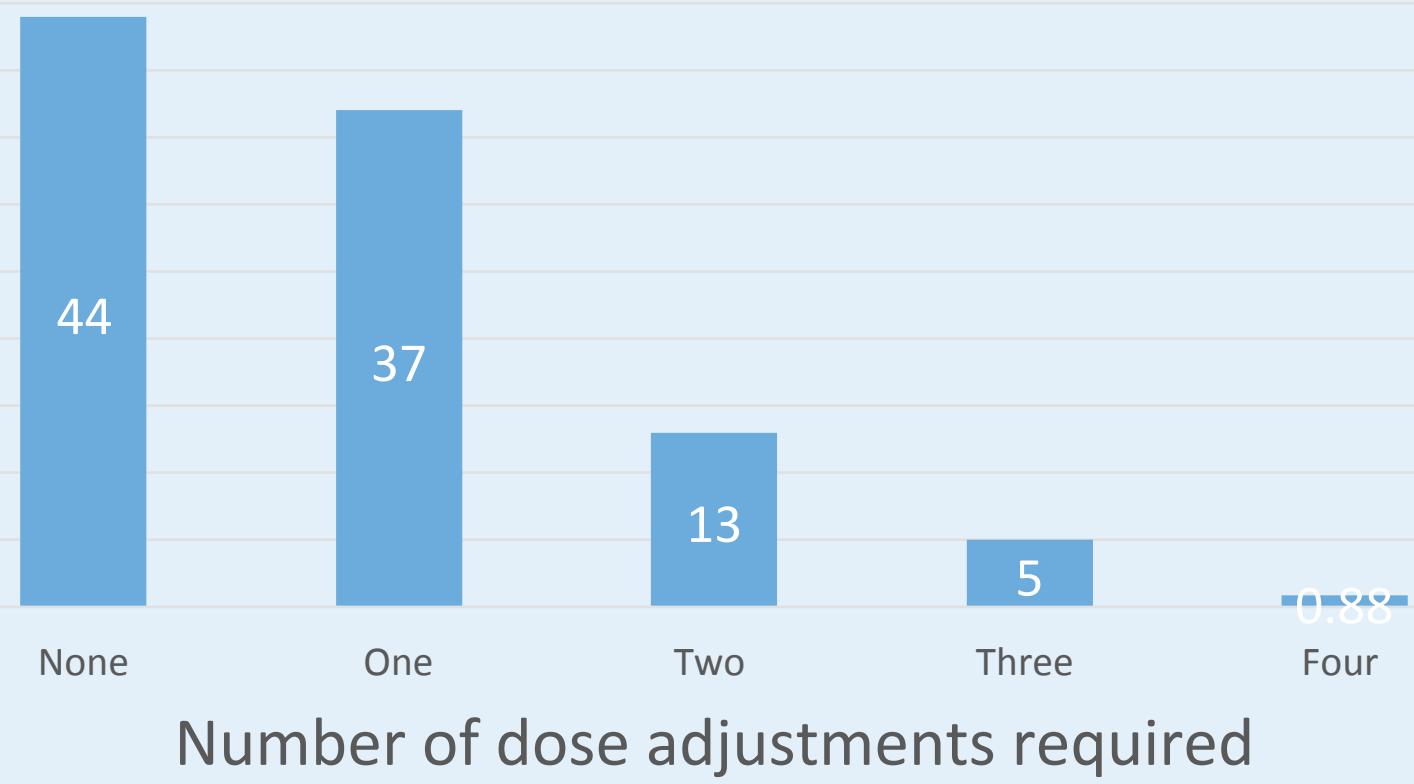

\section{Conclusions}

- As seen in the general population, hypothyroidism is the most common aetiology of thyroid disease

- The virtual clinic system is able to competently manage women with both preexisting and new-onset thyroid disease in pregnancy with no adverse outcomes

- Data shows that almost half of patients with pre-existing hypothyroidism do not require any dose adjustments during pregnancy; in those that do require an increase, the average is $41 \mathrm{mcg}(+/-19)$ - a $41 \%$ increase from the average initial dose

- This contrasts with current recommendations: are women with thyroid disease being over-medicated?

\section{Recommendations and}

\section{future practice}

- More research required in disease management regarding changes made to treatment throughout pregnancy with comparison to current recommendations:

$>$ This team will collect more data in order to compare two populations with contrasting patient demographics and evaluate any differences in aetiology or disease management [results expected early 2016]

$>$ Effects of ethnicity and thyroid antibody

- Evaluate relationship between baseline dose and likelihood of requiring dose adjustments through pregnancy - is there potential that residual function could lead to fewer changes? 